\title{
To Evaluate the Damage of Renal Function in CIAKI Rats at 3T: Using ASL and BOLD MRI
}

\author{
Wen-bo Chen, ${ }^{1,2}$ Long Liang, ${ }^{1,3}$ Bin Zhang, ${ }^{1,3}$ Chun-ling Liu, ${ }^{1}$ Hong-jun Liu, ${ }^{1}$ Hai-ying Luo, \\ Qiong-xin Zeng, ${ }^{1}$ Chang-hong Liang, ${ }^{1}$ Guan-shu Liu, ${ }^{4,5}$ and Shui-xing Zhang ${ }^{1,3}$ \\ ${ }^{1}$ Department of Radiology, Guangdong Academy of Medical Sciences/Guangdong General Hospital, No. 106 Zhongshan Er Road, \\ Guangzhou, Guangdong Province 510080, China \\ ${ }^{2}$ Department of Radiology, HuiZhou Municipal Central Hospital, No. 41 E Ling Bei Road, Huizhou, \\ Guangdong Province 516008, China \\ ${ }^{3}$ Southern Medical University, No. 1838 Dadao Bei Road, Guangzhou, Guangdong Province 510515, China \\ ${ }^{4}$ F. M. Kirby Research Center for Functional Brain Imaging, Kennedy Krieger Institute, Baltimore, MD, USA \\ ${ }^{5}$ The Russell H. Morgan Department of Radiology and Radiological Science, Division of MR Research, \\ Johns Hopkins University School of Medicine, Baltimore, MD, USA
}

Correspondence should be addressed to Shui-xing Zhang; shui7515@126.com

Received 12 December 2014; Accepted 15 March 2015

Academic Editor: Enzo Terreno

Copyright (C) 2015 Wen-bo Chen et al. This is an open access article distributed under the Creative Commons Attribution License, which permits unrestricted use, distribution, and reproduction in any medium, provided the original work is properly cited.

\begin{abstract}
Purpose. To investigate noninvasive arterial spin-labeling (ASL) and blood oxygen level-dependent imaging (BOLD) sequences for measuring renal hemodynamics and oxygenation in contrast induced acute kidney injury (CIAKI) rat. Materials and Methods. Thirteen SD rats were randomly grouped into CIAKI group and control group. Both ASL and BOLD sequences were performed at $24 \mathrm{~h}$ preinjection and at intervals of $0.5,12,24,48,72$, and $96 \mathrm{~h}$ postinjection to assess renal blood flow (RBF) and relative spin-spin relaxation rate $\left(R_{2}{ }^{*}\right)$, respectively. Results. For the CIAKI group, the value of RBF in the cortex (CO) and outer medulla (OM) of the kidney was significantly decreased $(P<0.05)$ at $12-48 \mathrm{~h}$ and regressed to baseline level $(P=\mathrm{NS})$ at $72-96 \mathrm{~h}$. In OM, the value of $R_{2}{ }^{*}$ was increased at $0.5-48 \mathrm{~h}(P<0.05)$ and not statistically significant $(P=\mathrm{NS})$ at 72 and $96 \mathrm{~h}$. Conclusions. RBF in OM and $\mathrm{CO}$ and oxygen level in OM were decreased postinjection of CM. ASL combining BOLD can further identify the primary cause of the decrease of renal oxygenation in CIAKI. This approach provides means for noninvasive monitoring renal function during the first 4 days of CIAKI in clinical routine work.
\end{abstract}

\section{Introduction}

Contrast induced acute kidney injury (CIAKI), used to be named as contrast-induced nephropathy (CIN), is a common complication in renal function mostly caused by the administration of contrast media $[1,2]$. And it is the most common cause of iatrogenic, inpatient, and drug-induced acute kidney injury (AKI) and the third commonest cause of hospital-acquired renal failure with an incidence of $11 \%$ [3-5]. The European Society of Urogenital Radiology (ESUR) Contrast Media Safety Committee proposed the definition of CIAKI as a condition in which a decrease in renal function occurs within 3 days after the intravascular administration of contrast media $(\mathrm{CM})$ without an alternative aetiology.
The cause and mechanism of CIAKI were various, but the three universally acknowledged causes may include kidney arteriolar vasoconstriction resulted from sustained contrastinduced hypoxia in cortex and medulla and the ischemiamediated oxidative stress causing renal damage [6]. Although many specialists hold that the high-osmolar should be the arch criminal for CIAKI, some studies made a point that lowosmolar and iso-osmolar CM resulted in the same incidence of CIAKI $[7,8]$.

Nuclear medicine and traditional MR perfusion weighted imaging were universally acknowledged to be useful for measuring kidney perfusion, but both were invasive and required intravenous injection. In recent years, blood flow and tissue oxygen bioavailability, which are founded to be 
changed in CIAKI as compared to baseline, can be measured noninvasively with MRI techniques called arterial spin labeling (ASL) and blood oxygen level-dependent (BOLD) MRI that does not require using additional markers or tracers [9-14]. Noninvasive ASL and BOLD MR techniques were demonstrated useful to reveal long-term damage in kidney function caused by ionic high-osmolality diatrizoate, particularly in outer medulla [15]. The comprehension of how blood flow and oxygenation change in the early phase has a high clinical relevance for monitoring the transient kidney injury during the course of administration of ionic iodinated contrast agent and evaluating the possibility and extent of induced permanent damage in kidney function. Towards this end, we adapted ASL and BOLD MRI methods for measuring renal hemodynamics and oxygenation in CIAKI rats up to 96 hours after the injection of CM using a 3 Tesla human scanner. Such an approach can be easily expanded to other renal diseases.

\section{Materials and Methods}

2.1. Animals. Thirteen male Sprague-Dawley (SD) rats with body weights of 200-250 g were obtained from provincial center for experimental animals. All animals were kept under standard conditions and fed with standard rodent chow and free water.

2.1.1. Ethics. All procedures were not only approved by the local Research Ethics Committee, but also were in accordance with the Guide of the Care and Use of Laboratory Animals published by the US National Institutes of Health (NIH Publication No. 85-23, revised 1996).

2.1.2. Animal Preparation. ALL the rats were injected to receive ionic iodinated contrast agent (Meglumine Diatrizoate, $370 \mathrm{mg} / \mathrm{mL}, 6 \mathrm{~mL} / \mathrm{kg}$ ) [15] via tail vein injection to induce CIAKI, after six untreated rats of them were scanned as the control. Each rat was fasted for 4 hours before MRI scans. Twenty minutes prior to MRI studies, the rat was anaesthetized with an intraperitoneal injection of $20 \%$ urethane $(6 \mathrm{~mL} / \mathrm{kg}$ b.w.). During anesthesia, rectal temperature was monitored to maintain the body temperature at $37 \pm 0.5^{\circ} \mathrm{C}$ using a heating lamp.

2.2. MR Studies. All experiments were performed using a 3.0T whole-body system (Signa EXCITE HD, GE Healthcare, Milwaukee, WI) with a $40 \mathrm{mT} / \mathrm{m}(200 \mathrm{mT} / \mathrm{m}-\mathrm{ms})$ gradient system and a transmit birdcage body coil with $50-\mathrm{mm}$ outer diameter and an eight channel receive-only volumetric rat array (RAPID Biomedical GmbH, Rimpar, Germany), for homogeneous RF transmission and signal detection respectively. The rats were placed head first and supine to make the kidneys in the RF center of the rat array coil. To determine the dynamic change of intrarenal perfusion and oxygenation, the CIAKI rats were scanned $0.5,12,24,48,72$ and $96 \mathrm{~h}$ after the injection of CM. At each time point, we got blood samplings from the postcava of the rats to examine renal function.

The details of the pulse sequences used are listed below.
(1) FSE-XL/T1WI Sequence. Axial Scan Mode = 2D, Grad Mode $=$ zoom, $\mathrm{TR}=500 \mathrm{~ms}, \mathrm{TE}=13.3 \mathrm{~ms}$, Flip Angle $=90^{\circ}$, Bandwidth $=15.6 \mathrm{~Hz}, \mathrm{NEX}=4.0, \mathrm{FOV}=$ $10.0 \times 7.0 \mathrm{~cm}$, Slice Thickness $=2.0 \mathrm{~mm}$, Sap $=0.2 \mathrm{~mm}$, Matrix $=288 \times 192, \mathrm{ET}=3$, and Scan Time $=94 \mathrm{~s}$.

(2) FSE-XL/T2WI Sequence. Axial and coronal Scan Mode $=2 \mathrm{D}$, Grad Mode $=$ zoom, $\mathrm{TR}=4500 \mathrm{~ms}, \mathrm{TE}=$ $125.8 \mathrm{~ms}$, Bandwidth $=62.50 \mathrm{~Hz}, \mathrm{NEX}=6.0, \mathrm{FOV}=$ $10 \times 7.5 \mathrm{~cm}$, Slice Thickness $=2.0 \mathrm{~mm}$, Sap $=0.2 \mathrm{~mm}$, Matrix $=288 \times 192, \mathrm{ET}=19$, and Scan Time $=443 \mathrm{~s}$.

(3) ASL-FAIR-SSFSE Sequence. Coronal Scan Mode =2D, Grad Mode $=$ zoom, $\mathrm{TR}=2453 \mathrm{~ms}, \mathrm{TE}=119.8 \mathrm{~ms}$, Bandwidth $=83.3 \mathrm{~Hz}, \mathrm{FOV}=10.0 \times 10.0 \mathrm{~cm}$, Slice Thickness $=2.0 \mathrm{~mm}$, Sap $=0.2 \mathrm{~mm}$, Matrix $=128 \times$ $128, \mathrm{ET}=19$, Multislice $=4$ slices, Scan Time $=159 \mathrm{~s}$.

(4) BOLD-MFGRE Sequence: Coronal Scan Mode = 2D, Grad Mode $=$ zoom, $\mathrm{TR}=100 \mathrm{~ms}, \mathrm{TE}=4.0 \mathrm{~ms}$, Bandwidth $=31.3 \mathrm{~Hz}$, FOV $=10.0 \times 10.0 \mathrm{~cm}$, Slice Thickness $=2.2 \mathrm{~mm}$, Sap $=0.2 \mathrm{~mm}$, Matrix $=96 \times 96$, $\mathrm{ET}=19$, Multislice $=4$ slices, Scan Time $=78 \mathrm{~s}$.

\subsection{Postprocessing}

2.3.1. ASL. A four-layer acquisition was performed on both kidneys, with 20 different images at each layer. All images were used to measure renal perfusion by functool-fair software on Advantage Workstation 4.3 GE Medical System (AW4.3_05).

2.3.2. BOLD. Parametric images of $T_{2}{ }^{*}$ and $R_{2}{ }^{*}$ (equal to $\left.1 / T_{2}{ }^{*}\right)$ were obtained on a pixel-by-pixel basis using the Advantage Workstation 4.3 GE Medical System (AW4.3_05). Measurements of $R_{2}{ }^{*}$ and $T_{2}{ }^{*}$ were obtained including cortex (CO), outer medulla (OM) and inner medulla (IM). The slope of natural logarithm of signal intensity versus echo time equals to relaxation rate $R_{2}{ }^{*}$ which is positive correlation with the concentration of deoxyhemoglobin.

All MR images were evaluated by two radiologists blindly to the group designations. The two radiologists were asked to reach a consensus if there was a discrepancy in the result. The regions of interest (ROIs) were selected at the level of renal hilum of the kidney, with the same size and shape (Figure 1). Each ROI had an area of $3.5-5.5 \mathrm{~mm}^{2}$ and contained at least five pixels. ROI was measured in cortex, outer medulla, and inner medulla, without any distortions, artifacts, large vessels, renal collecting system or any incidental renal cysts in the ROIs.

2.4. Biochemistry Assessment. The blood collected from the inferior vena cava of rats was centrifuged at the speed of 3500 rmp for $10 \mathrm{~min}$. The obtained serum was measured for the serum creatinine concentration, which reflects the injury of kidney function in the model rats.

2.5. Statistical Analysis. Data were analyzed using SPSS 13.0 for Windows. $K$ independent samples test and MannWhitney $U$ test for further comparisons between specific 


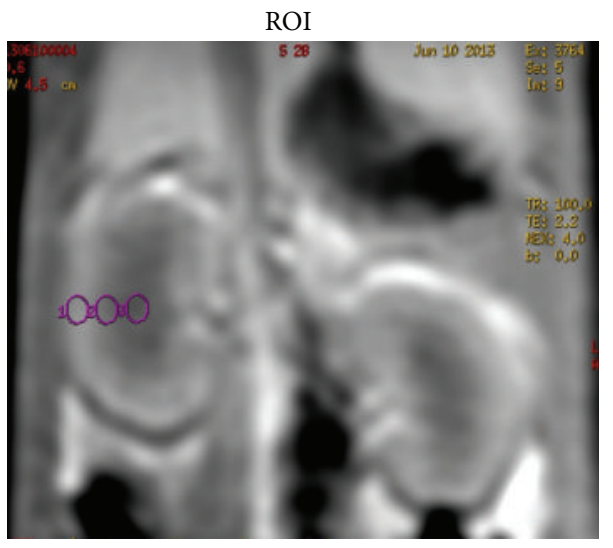

(a)

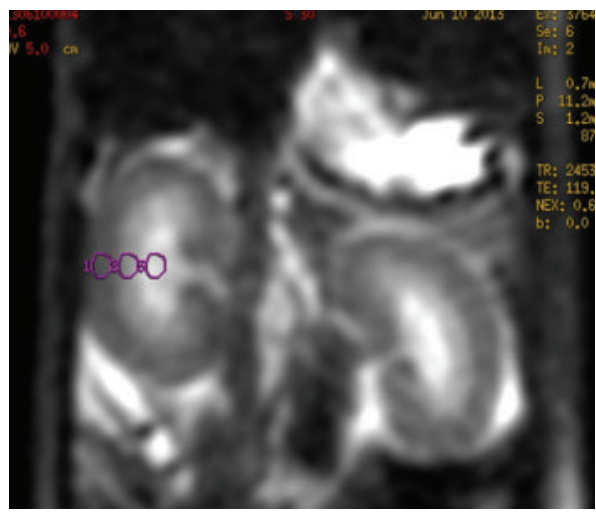

(c)

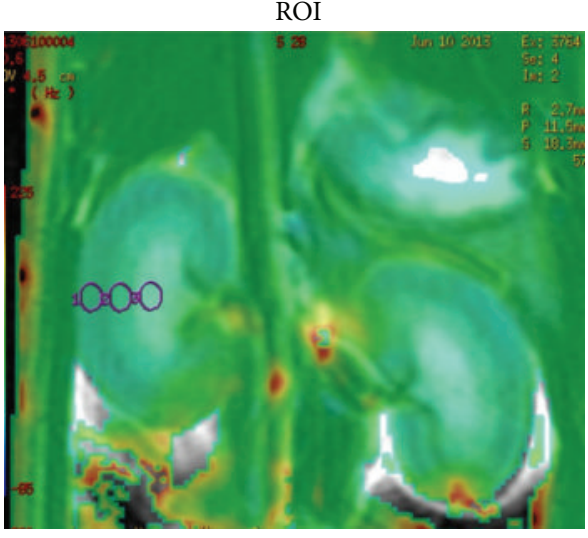

(b)

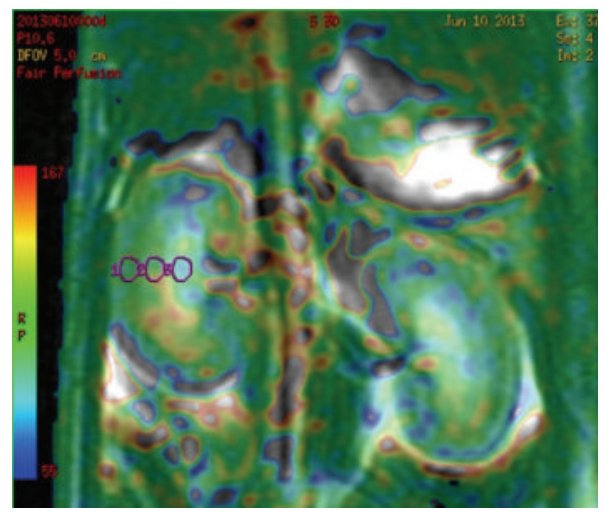

(d)

FIGURE 1: Representative coronal MR image (a) and the $R_{2}{ }^{*}$ map (b) acquired using a BOLD sequence. Representative coronal MR image (c) the RBF map (d) acquired using an ASL sequence. The ROI putting on each kidney structure assembles the CO (1), OM (2), and IM (3). In (b) and (d), function parametric maps were overlaid on the top of the corresponding coronal MR image. Software automatically sums the number of voxels, MR signal intensity and calculate the RBF and $R_{2}{ }^{*}$ values.

TABLE 1: RBF value of pre- and postinjection of CM $\left(\mathrm{mL} \cdot 100 \mathrm{~g}^{-1} \cdot \mathrm{min}^{-1}\right)$.

\begin{tabular}{lccc}
\hline Group & Cortex & Outer-medulla & Inner-medulla \\
\hline Baseline $(n=6)$ & $120.84 \pm 2.34$ & $109.26 \pm 4.17$ & $111.36 \pm 5.22$ \\
$30 \min (n=6)$ & $110.62 \pm 18.78$ & $99.84 \pm 8.95$ & $107.36 \pm 18.13$ \\
$12 \mathrm{~h}(n=6)$ & $97.89 \pm 3.69^{*}$ & $95.37 \pm 3.74^{*}$ & $109.32 \pm 20.41$ \\
$24 \mathrm{~h}(n=6)$ & $97.35 \pm 3.51^{*}$ & $93.62 \pm 2.20^{*}$ & $108.56 \pm 11.85$ \\
$48 \mathrm{~h}(n=6)$ & $93.19 \pm 2.64^{*}$ & $94.11 \pm 6.31^{*}$ & $107.36 \pm 8.39$ \\
$72 \mathrm{~h}(n=6)$ & $105.83 \pm 16.79$ & $100.10 \pm 17.29$ & $91.66 \pm 20.88$ \\
$96 \mathrm{~h}(n=3)$ & $106.15 \pm 16.1$ & $103.41 \pm 11.51$ & $104.17 \pm 5.55$ \\
\hline
\end{tabular}

$K$ independent samples test, respectively, and Mann-Whitney $U$ test for further comparisons between specific group pairs. ${ }^{*} P<0.05$.

group pairs were used. $P<0.05$ was used as the criteria for the statistical difference among groups.

\section{Results}

Except for one rat which died at 30 min after intravascular administration of Meglumine Diatrizoate, likely due to overdose of $20 \%$ urethane, all other twelve rats completed the entire protocol including the overall ASL imaging and BOLD imaging successfully until $72 \mathrm{~h}$ postinjection Besides, there were anther 3 rats died before $96 \mathrm{~h}$ time point MRI scan likely due to fasting and narcotized over and over again at each time point and resulted in a poor condition and even died. All the data were successfully recorded. Both the RBF values and $R_{2}{ }^{*}$ values could be calculated from each time point map.

The spatial resolution (i.e., $0.78 \times 0.78 \mathrm{~mm}$ ) of all the ASL maps used for RBF measurement was good enough to distinguish among the $\mathrm{CO}, \mathrm{OM}$, and IM of the kidney (Figure 2). The RBF values in the CO, OM and IM in the rats before and after the injection of iodinated contrast agent were shown in Table 1. In the CO and OM of the kidney, the values of RBF at 12,24 , and $48 \mathrm{~h}$ after injection were found 
TABLE 2: $R_{2}{ }^{*}$ value of pre- and postinjection of $\mathrm{CM}($ mean $\pm \mathrm{SD} ; \mathrm{Hz})$.

\begin{tabular}{lccr}
\hline Group & Cortex & Outer-medulla & Inner-medulla \\
\hline Baseline $(n=6)$ & $29.09 \pm 3.22$ & $26.12 \pm 1.92$ & $26.03 \pm 4.27$ \\
$30 \min (n=6)$ & $36.58 \pm 7.09$ & $39.37 \pm 4.57^{*}$ & $36.36 \pm 9.84$ \\
$12 \mathrm{~h}(n=6)$ & $33.99 \pm 4.27$ & $39.86 \pm 4.37^{*}$ & $38.57 \pm 13.01$ \\
$24 \mathrm{~h}(n=6)$ & $35.90 \pm 7.61$ & $38.85 \pm 2.75^{*}$ & $29.54 \pm 5.68$ \\
$48 \mathrm{~h}(n=6)$ & $36.59 \pm 8.34$ & $40.94 \pm 4.23^{*}$ & $36.29 \pm 7.12$ \\
$72 \mathrm{~h}(n=6)$ & $29.48 \pm 2.30$ & $32.39 \pm 2.89$ & $30.03 \pm 4.70$ \\
$96 \mathrm{~h}(n=3)$ & $31.22 \pm 2.67$ & $24.98 \pm 6.99$ & $22.91 \pm 9.06$ \\
\hline
\end{tabular}

$K$ independent samples test, respectively, and Mann-Whitney $U$ test for further comparisons between specific group pairs. ${ }^{*} P<0.05$.

ASL

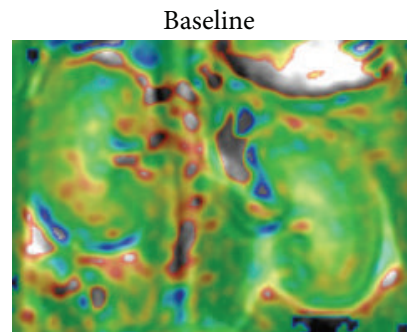

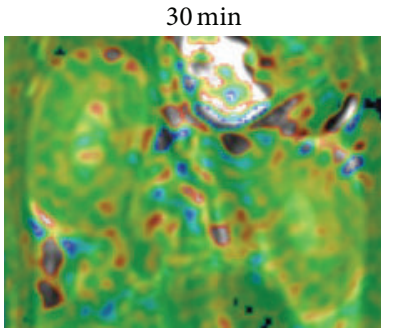

$12 \mathrm{~h}$
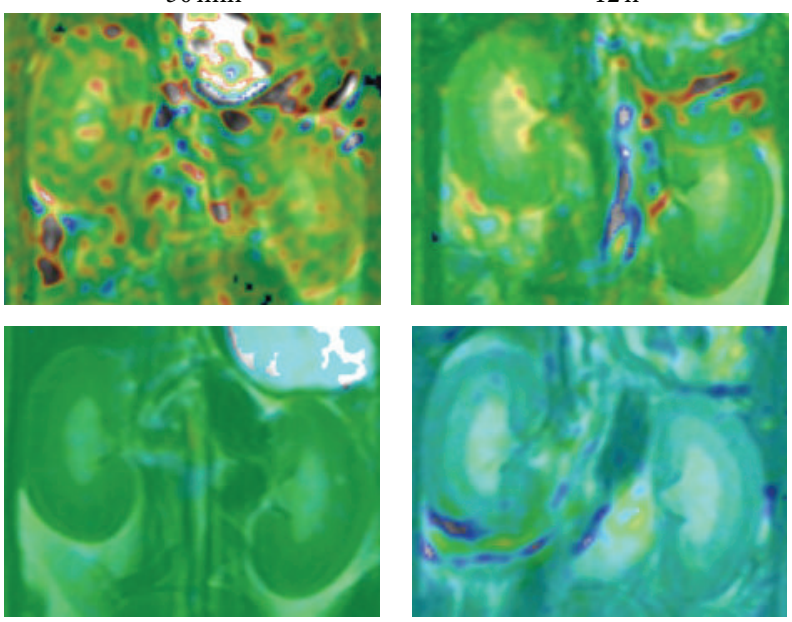

$72 \mathrm{~h}$

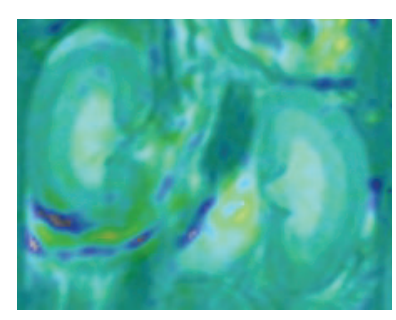

$96 \mathrm{~h}$

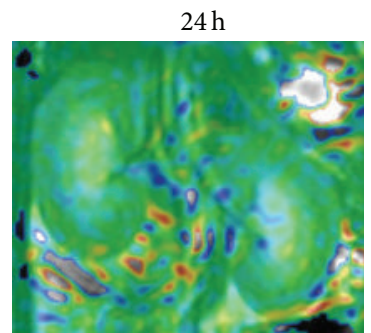

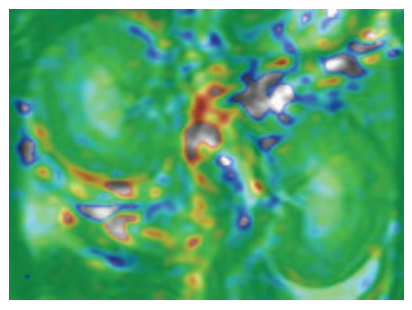

$48 \mathrm{~h}$

FIGURE 2: Time course of RBF changes in the kidney of a representative CIAKI rat over a $96 \mathrm{~h}$ period after injection of iodinated CM using an ASL sequence. Representative coronal SSFSE MR maps include CO, OM, and IM of each kidney before and 0.5, 12, 24, 48, 72 and 96 h after infusion.

significantly decreased $(P<0.05$ versus baseline, $n=6$, resp.; Figures 3 and 4 ), while those at 72 and 96 h gradually regressed to a level close to baseline ( $P=$ NS versus baseline).

All the BOLD images also had enough spatial resolution (i.e., $1 \times 1 \mathrm{~mm}$ ) to distinguish $\mathrm{CO}$, OM and IM of the kidneys (Figure 5). Table 2 shows the average change of oxygen level in the regions of $\mathrm{CO}, \mathrm{OM}$ and IM in the CIAKI rats. In the kidney OM, the value of $R_{2}{ }^{*}$ were greatly increased between $30 \mathrm{~min}$ to $48 \mathrm{~h}(P<0.05$ versus baseline, $n=6$, Figure 6$)$, but fell back to baseline after $72 \mathrm{~h}(P=$ NS versus baseline). The CM did not produce statistically significant changes in $R_{2}{ }^{*}$ within the kidney CO and IM ( $P=$ NS versus baseline).

The value of $\mathrm{RBF}$ and $R_{2}{ }^{*}$ were also assessed using the exact same procedure in six untreated rats before CM injection. In addition, there was no statistical significance in the mean $\mathrm{RBF}$ and $R_{2}{ }^{*}$ during the time course of our study, proving that the changes we found in the CIAKI rats using ASL and BOLD methods were the direct consequences of kidney injury.

To further verify whether kidney was injury after injection of iodinated CM and allows correlation with disease severity, blood serum creatinine was measured. The serum creatinine concentration reached the maximum at $72 \mathrm{~h}(P<$ 0.05 versus baseline, Figure 7$)$, whereas at other time points the difference did not reach statistical significance $(P=\mathrm{NS}$ versus baseline). As shown in Figure 8, the changes of serum creatinine value were relatively later than both the changes in $R_{2}{ }^{*}$ value and $\mathrm{RBF}$ value.

\section{Discussion}

CIAKI was defined as the decrease in renal function after CM injection, using SCr as a marker. However, SCr is a surrogate marker for glomerular filtration and does not provide the complete information of kidney function. For example, it was reported that when the impaired nephrons is less than $50 \%$, no change or only marginal change of SCr could be observed [2]. Therefore a comprehensive method that describes the whole spectrum of kidney function in CIAKI is highly desirable. As previous study [15] reported that ASL and BOLD MRI were useful to reveal renal damage caused by ionic high-osmolality diatrizoate, our study tried to use this two MRI techniques to monitor the dynamic change of renal function in CIAKI model. 


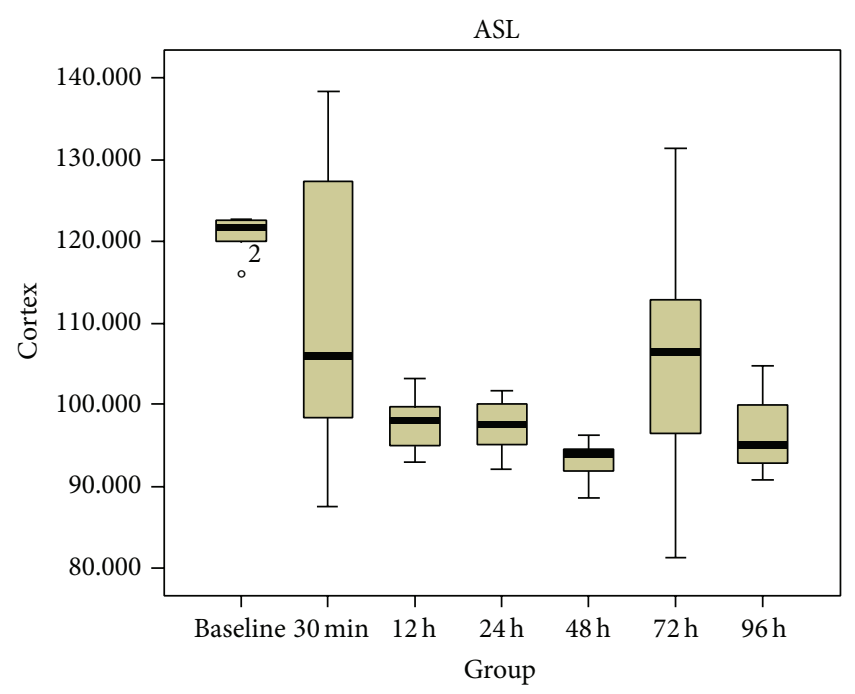

FIgure 3: Box plots of RBF values in CO. The top and bottom of the boxes indicate the first and third quartiles, respectively. The length of box represents the interquartile range, within which $50 \%$ of the values were located. The solid line within each box is the median. The error bars show the minimum and maximum values (range). The circle mean the outlier. This box plot implied that the $50 \%$ of the values at $12 \mathrm{~h}, 24 \mathrm{~h}, 48 \mathrm{~h}$ were lower than baseline.

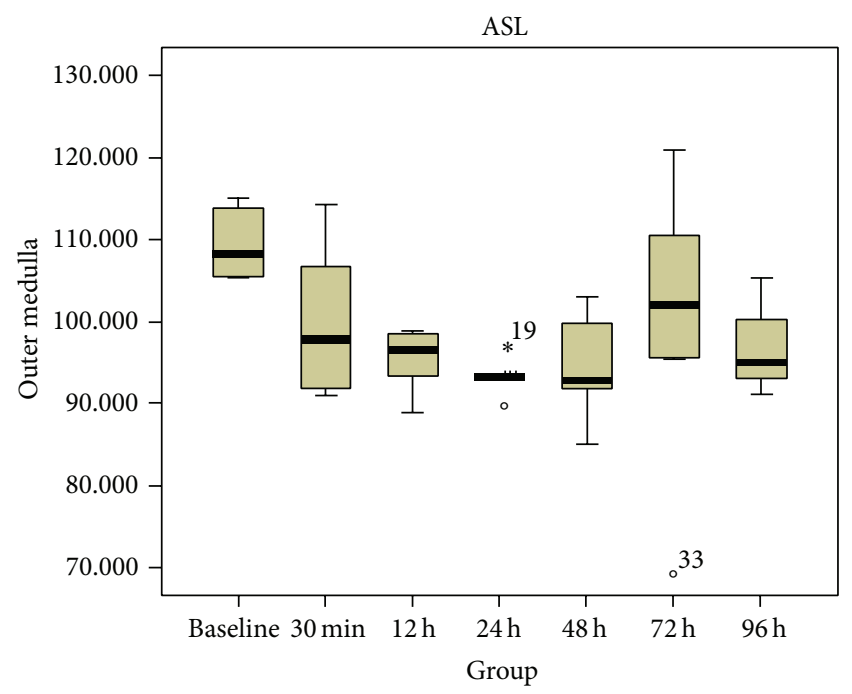

FIgURE 4: Box plots of RBF values in OM. The circle and asterisk mean the outlier and extremum respectively. This box plot implied that the $50 \%$ of the values at $12 \mathrm{~h}, 24 \mathrm{~h}, 48 \mathrm{~h}$ were lower than baseline.

In order to measure the kidney function more accurately, we have made several improvements of MR scan. In particular, we implemented a pulse sequence to measurement both pre- and postinjection CM. And we also utilized a free breathing protocol acquisition. In addition, we chose a coronal orientation to avoid major feeding vessels. With these improvements, we measured the time-evolution of RBF and $R_{2}{ }^{*}$ value in both intact and CIAKI kidneys over a broad range of function.
Our results demonstrated a significant decrease of RBF value in both $\mathrm{CO}$ and $\mathrm{OM}$, which is similar to previous reports [16]. But it should be noted that the RBF value of CO in intact animals obtained in our study are slightly lower than those reported by Liu et al. [16], (i.e., baseline level our $\mathrm{RBF}$ value was $120.84 \pm 2.34 \mathrm{~mL} / 100 \mathrm{~g} / \mathrm{min}$ versus $124.89 \pm$ $6.60 \mathrm{~mL} / 100 \mathrm{~g} / \mathrm{min} ; 24 \mathrm{~h}$ group our RBF value was $97.35 \pm$ $3.51 \mathrm{~mL} / 100 \mathrm{~g} / \mathrm{min}$ versus $123.49 \pm 9.16 \mathrm{~mL} / 100 \mathrm{~g} / \mathrm{min}$ ). This may partly be due to the variation in acquisition and processing.

Some studies about CIAKI focused on the acute effects of iodinated $\mathrm{CM}$ on renal hemodynamic distribution (i.e., from the first hour after CM injection to $24 \mathrm{~h}$ ) $[15,17,18]$. Our study revealed a prolonged decrease of RBF value in both $\mathrm{CO}$ and OM last to $48 \mathrm{~h}$ after the injection of diatrizoate, and gradually returned to a level close to baseline at later time (72 and $96 \mathrm{~h}$ post-injection). As we know, signal intensity on FAIR-ASL is attributed to blood flow including both artery and vein. Therefore, a persistent stasis-state of venous blood cells after iodinated CM injection may probably increase the cardiac afterload and decrease the blood speed and renal perfusion, which must have an important contribution in the decease of RBF value [19]. And acute vasoconstriction in the renal capillaries caused by the accumulation of the injected iodinated agents may be the deeper reason. Besides, the mechanism why RBF value decreased, especially in the outer medulla, could be explained as follows. Firstly, CM could directly decrease blood flow of renal medullary by increasing plasma viscosity. Secondly, CM with high concentration could cause a pronounced osmotic diuresis that distends the tubules and collecting ducts, leading to renal swelling, increasing intrarenal venous pressure [20] and decreasing outer medullary blood flow. Taking account of persistent stasis-state of venous blood cells into consideration, it can be explained why the RBF value of OM was the lowest among the kidney structures measured.

Originating from the $T_{2}{ }^{*}$ effect of oxyhemoglobin/deoxyhemoglobin, the $R_{2}{ }^{*}$ in tissues measured by BOLD negatively correlates with the tissue content of oxygen $\left(\mathrm{PO}_{2}\right)$ [21]. On CIAKI rats, from $30 \mathrm{~min}$ to $48 \mathrm{~h}$, our data demonstrated a significant increase in $R_{2}{ }^{*}$ only in OM of kidney using BOLD MRI, indicating a decrease of oxygen level, which was agreed with other studies [22]. Several mechanisms can be attributed to this result. First, as diatrizoate is a hyperosmotic $\mathrm{CM}$, the osmotic effect of agent could elevate the amount of sodium collected at the thick ascending limb of the loop of Henle, which in turn accelerate the active transportation of cations from the loop of Henle [23] and cause an increase of oxygen utilization. Second, due to the high viscosity of the circulating $\mathrm{CM}$, the aggregation of red blood cells may occur, resulting in a decrease of oxygen delivery indirectly by slowing intrarenal blood flow $[24,25]$. Finally, iodinated CM could induce medullary vasoconstriction, directly resulting in renal hypoxia $[26,27]$. Besides, $R_{2}{ }^{*}$ can also be influenced by many pathological conditions, such as iron concentration, the oxygenation ability of hemoglobin, or hematocrit. But in our study, we measured the $R_{2}{ }^{*}$ value at different postinjection times as compared to that preinjection from the same rat, in which iron concentration, the oxygenation ability of 

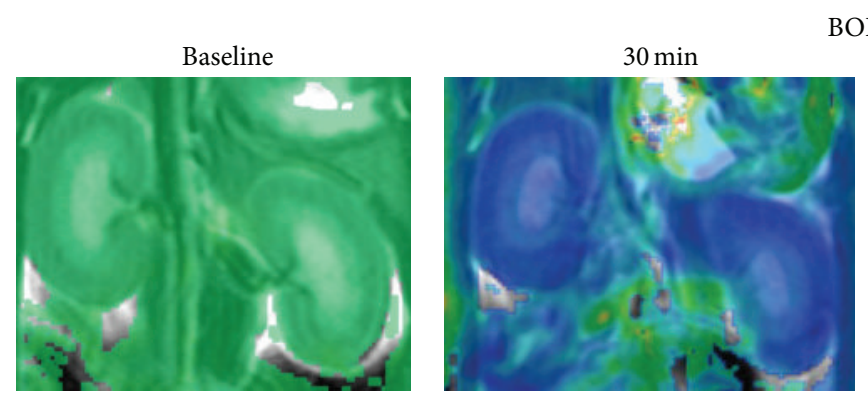

BOLD

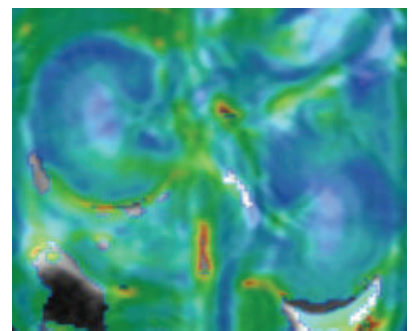

$48 \mathrm{~h}$

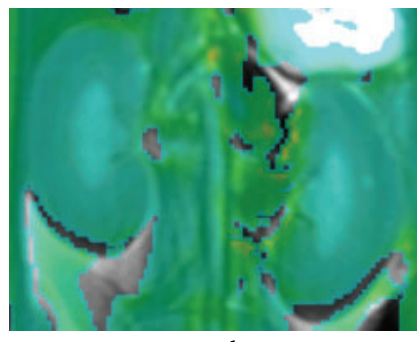

$72 \mathrm{~h}$
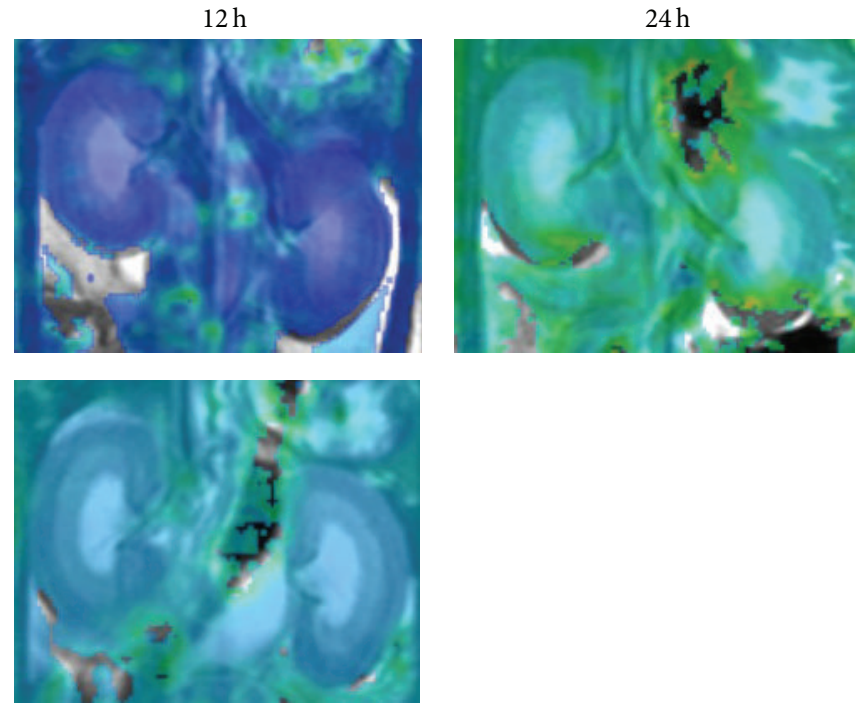

$96 \mathrm{~h}$

FIGURE 5: Time course studies in BOLD sequence of baseline and CIAKI rats over a $96 \mathrm{~h}$ period after injection of iodinated CM. Representative coronal MFGRE MR maps include CO, OM, and IM of each kidney before and at 0.5, 24, 48, 72 and $96 \mathrm{~h}$ after CM injection.

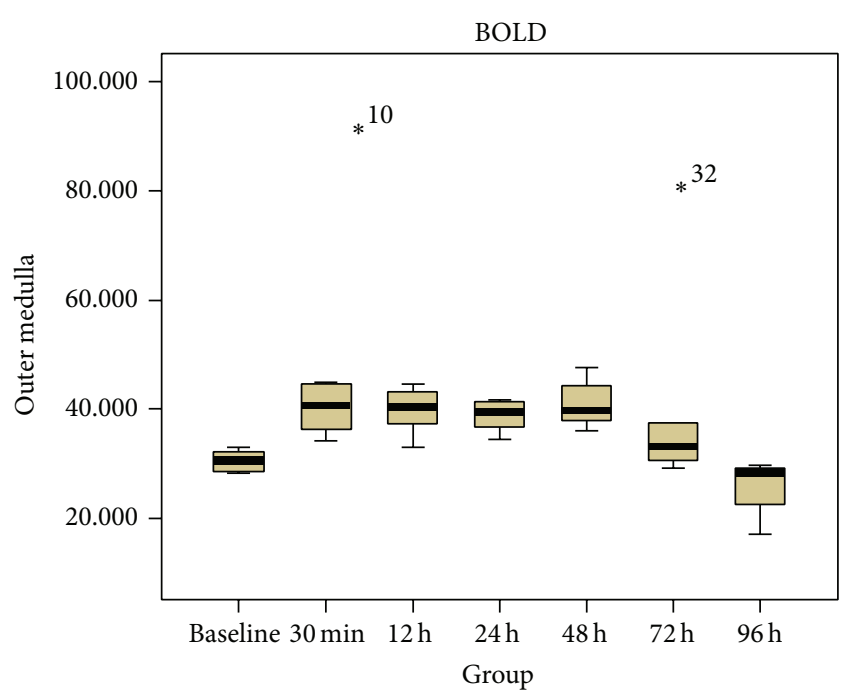

Figure 6: Box plots of $R_{2}{ }^{*}$ values in OM. The asterisk means the extremum. This box plot demonstrate that the $50 \%$ of the values at $0.5,24$ and $48 \mathrm{~h}$ were larger than baseline, while at $72 \mathrm{~h}$ and $96 \mathrm{~h}$ time point the overlap was present with baseline.

hemoglobin, or hematocrit is likely to be on the same level. By this way, we wanted to minimize the influence of alternative causes to the $R_{2}{ }^{*}$.

What is more, the purpose of why we chose ASL combined with BOLD in monitoring renal damage was to help further identify whether the decrease of renal oxygenation was accounted for either the decrease of blood perfusion or the increase of oxygen consumption. In our study, the increase of $R_{2}{ }^{*}$ were greatly increased in $30 \mathrm{~min}$ after CM injection in OM, while the RBF was no difference in this time. Therefore we may identify in rough that the increase

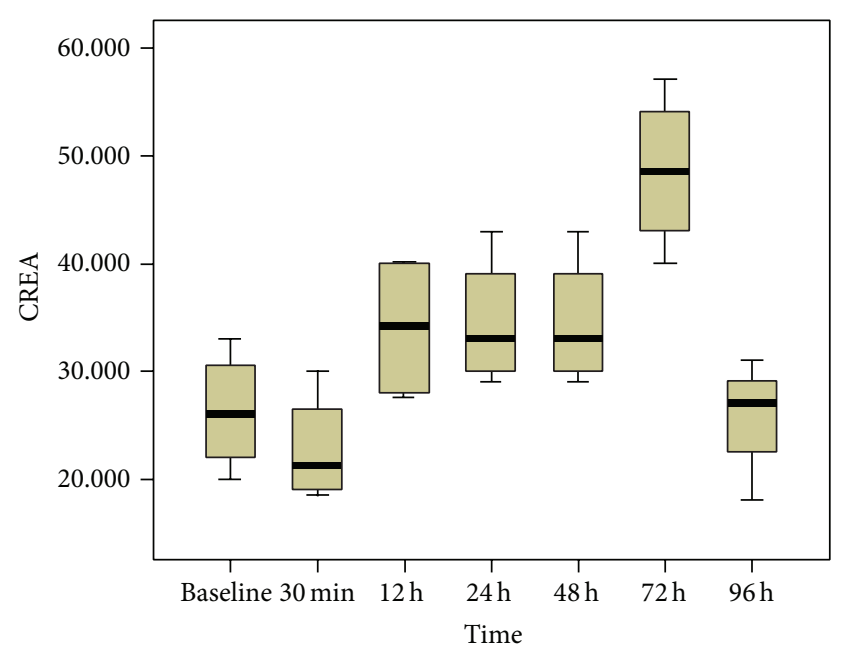

FIGURE 7: The concentration of serum creatinine before and $0.5,12$, $24,48,72$ and $96 \mathrm{~h}$ after injection of CM. The concentration of serum creatinine was significantly increased only at $72 \mathrm{~h}(P<0.05$ versus baseline), whereas at other time points the differences did not reach statistical significance ( $P=$ NS versus baseline).

of oxygen consumption played an more important role in the decrease of renal oxygenation.

In patients, SCr level commonly reaches the peak within 2-5 days after CM administration [28]. Our result demonstrated that the peak of SCr occurs at approximate 72 hours (3 days) after the CM injection, indicating the time course of kidney injury in our animal model is well correlated with patients. Our study implies that both the BOLD and ASL sequence might find the renal injury began no later than $12 \mathrm{~h}$, which was much earlier than blood serum creatinine changes. So, we might conclude that both ASL and BOLD techniques 


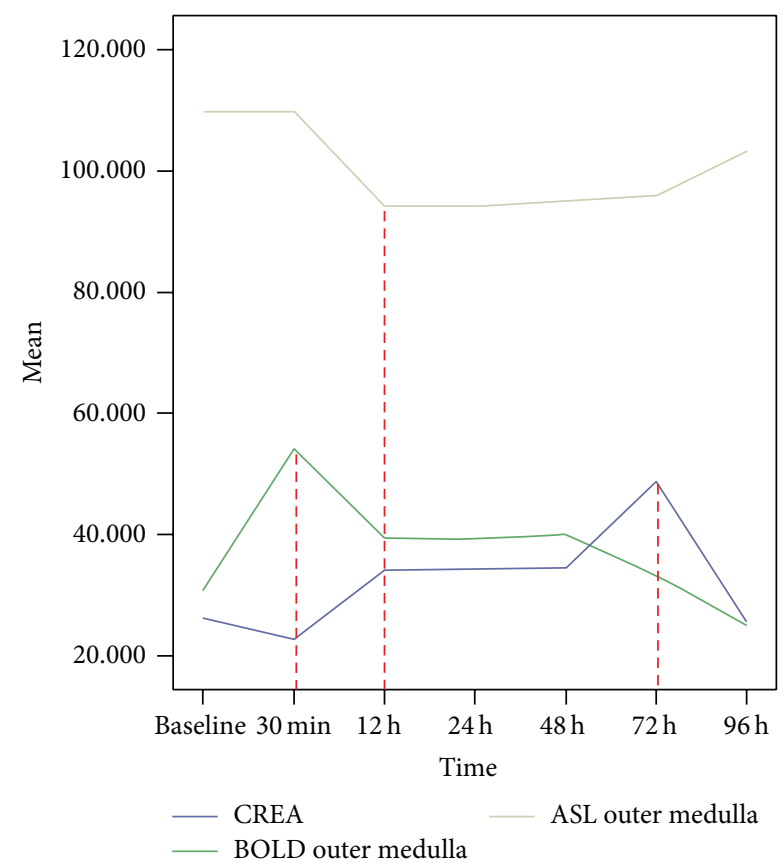

FIGURE 8: The $R_{2}{ }^{*}$ value, RBF value of OM and the concentration of serum creatinine. The superior line demonstrated that the RBF value was significantly decreased at the $12 \mathrm{~h}$ time point and last to $48 \mathrm{~h}$ $(P<0.05$ versus baseline). The value of middle line demonstrated $R_{2}{ }^{*}$ were greatly increased at $30 \mathrm{~min}$ and last to $48 \mathrm{~h}(P<0.05$ versus baseline). The inferior line demonstrated the concentration of serum creatinine was significantly increased only at $72 \mathrm{~h}(P<0.05$ versus baseline). The changes of serum creatinine values were relatively later than both the changes of $R_{2}{ }^{*}$ value and RBF value.

were much more sensitive than SCr in detecting the renal injury after CM injection.

On untreated animals, variation of both RBF and $R_{2}{ }^{*}$ values, either among the different kidney structures or over time in the same structure, were small, indicating ASL and BOLD techniques used in our study are reliable for monitoring the damage caused by $\mathrm{CM}$ injection, as well as other kidney diseases. As these two MR techniques have been much more widely used in some clinical regimes, our approach may be quickly translated to the patient study for monitoring the damage of renal function in a variety of kidney diseases, especially in CIAKI that we focus on here.

As discussed above we might conclude that ASL combining BOLD could provide much more convictive datas for revealing the primary cause of the decrease of renal oxygen $\left(\mathrm{PO}_{2}\right)$ in CIAKI. It would certainly help clinicians to give the right medicines to their CIAKI patients, by telling them which is more important between oxygen consumption and blood perfusion decrease.

\section{Clinical Application}

Evaluation of the damage of iodinated CM on kidney hemodynamics and oxygenation is critical for better comprehension and prevention of renal hypoxia diseases. BOLD and ASL MRI provide a rapid and accurate means for evaluation of kidney function of most of the common kidney diseases, such as CIAKI, diabetic nephropathy, and kidney transplant. In addition, these techniques can also be used to monitor the therapeutic responses of treatments to common renal hypoxia disease.

\section{Our Study Has Some Limitations}

Our study has several limitations, which may need future investigation. While we measured the serum creatinine level to validate our MRI findings, a histological analysis of renal morphology may be useful to identify the regions of injury. However, because both RBF and $R_{2}{ }^{*}$ change back to normal after 96 hours, the histological analysis after the final imaging time point may not be useful. Besides, our study did not take the effect of iodinated $\mathrm{CM}$ on the tissue $T_{1}$ relaxation into consideration because it took such a long time to measure kidney $T_{1}$ relaxation with an IR sequence. In the future study, we will continue to work on improving the accuracy of the measurement and the quality of images by refining our imaging protocol.

In conclusion, we have successfully employed noninvasive ASL and BOLD MRI to acquire high-quality images using 3.0T MRI clinical scanner, allowing the measurement of renal hemodynamics and oxygenation. Our results revealed a significant and prolonged decrease of RBF in OM and CO and also a decreased oxygen level in OM after CM injection, indicating that ASL and BOLD MRI are potentially useful in the clinic for noninvasive assessing of renal function in the case of CIAKI.

\section{Conflict of Interests}

The authors declare that there is no conflict of interests regarding the publication of this paper.

\section{Acknowledgments}

This work is partly supported by grants to Shui xing Zhang the National Scientific Foundation of China (Grant 81171329) and Guangdong science Foundation (Grant S2011010000790).

\section{References}

[1] L. Yin, G. Li, T. Liu et al., "Probucol for the prevention of cystatin C-based contrast-induced acute kidney injury following primary or urgent angioplasty: a randomized, controlled trial," International Journal of Cardiology, vol. 167, no. 2, pp. 426-429, 2013.

[2] C. Ronco, F. Stacul, and P. A. McCullough, "Subclinical acute kidney injury (AKI) due to iodine-based contrast media," European Radiology, vol. 23, no. 2, pp. 319-323, 2013.

[3] S. D. Weisbord, M. K. Mor, A. L. Resnick et al., "Prevention, incidence, and outcomes of contrast-induced acute kidney injury," Archives of Internal Medicine, vol. 168, no. 12, pp. 13251332, 2008.

[4] K. Nash, A. Hafeez, and S. Hou, "Hospital-acquired renal insufficiency," American Journal of Kidney Diseases, vol. 39, no. 5, pp. 930-936, 2002. 
[5] A. Linkermann, J.-O. Heller, Á. Prókai et al., “The RIP1kinase inhibitor necrostatin-1 prevents osmotic nephrosis and contrast-induced AKI in mice," Journal of the American Society of Nephrology, vol. 24, no. 10, pp. 1545-1557, 2013.

[6] P. A. McCullough, "Contrast-induced acute kidney injury," Journal of the American College of Cardiology, vol. 51, no. 15, pp. 1419-1428, 2008.

[7] M. Reed, P. Meier, U. U. Tamhane, K. B. Welch, M. Moscucci, and H. S. Gurm, "The relative renal safety of iodixanol compared with low-osmolar contrast media : a meta-analysis of randomized controlled trials," JACC: Cardiovascular Interventions, vol. 2, no. 7, pp. 645-654, 2009.

[8] P. A. McCullough and J. R. Brown, "Effects of intra-arterial and intravenous iso-osmolar contrast medium (iodixanol) on the risk of contrast-induced acute kidney injury: a meta-analysis," Cardiorenal Medicine, vol. 1, no. 4, pp. 220-234, 2011.

[9] C. de Bazelaire, D. C. Alsop, D. George et al., "Magnetic resonance imaging-measured blood flow change after antiangiogenic therapy with PTK787/ZK 222584 correlates with clinical outcome in metastatic renal cell carcinoma," Clinical Cancer Research, vol. 14, no. 17, pp. 5548-5554, 2008.

[10] N. Karger, J. Biederer, S. Lüsse et al., "Quantitation of renal perfusion using arterial spin labeling with FAIR-UFLARE," Magnetic Resonance Imaging, vol. 18, no. 6, pp. 641-647, 2000.

[11] N. F. Schwenzer, C. Schraml, P. Martirosian, A. Boss, C. D. Claussen, and F. Schick, "MR measurement of blood flow in the parotid gland without contrast medium: a functional study before and after gustatory stimulation," NMR in Biomedicine, vol. 21, no. 6, pp. 598-605, 2008.

[12] P. J. van Laar, J. van der Grond, and J. Hendrikse, "Brain perfusion territory imaging: methods and clinical applications of selective arterial spin-labeling MR imaging," Radiology, vol. 246, no. 2, pp. 354-364, 2008.

[13] D. S. Williams, J. A. Detre, J. S. Leigh, and A. P. Koretsky, "Magnetic resonance imaging of perfusion using spin inversion of arterial water," Proceedings of the National Academy of Sciences of the United States of America, vol. 89, no. 1, pp. 212216, 1992.

[14] J. Neugarten, "Renal BOLD-MRI and assessment for renal hypoxia," Kidney International, vol. 81, no. 7, pp. 613-614, 2012.

[15] Y. Zhang, J. Wang, X. Yang et al., "The serial effect of iodinated contrast media on renal hemodynamics and oxygenation as evaluated by ASL and BOLD MRI," Contrast Media \& Molecular Imaging, vol. 7, no. 4, pp. 418-425, 2012.

[16] Y. P. Liu, R. Song, C. H. Liang, X. Chen, and B. Liu, "Arterial spin labeling blood flow magnetic resonance imaging for evaluation of renal injury," American Journal of PhysiologyRenal Physiology, vol. 303, no. 4, pp. F551-F558, 2012.

[17] D. Rauch, P. Drescher, F. J. Pereira, J. M. Knes, J. A. Will, and P. O. Madsen, "Comparison of iodinated contrast media-induced renal vasoconstriction in human, rabbit, dog, and pig arteries," Investigative Radiology, vol. 32, no. 6, pp. 315-319, 1997.

[18] G. Deray, C. Bagnis, C. Jacquiaud, M. Dubois, Y. Adabra, and C. Jaudon, "Renal effects of low and isoosmolar contrast media on renal hemodynamic in a normal and ischemic dog kidney," Investigative Radiology, vol. 34, no. 1, pp. 1-4, 1999.

[19] E. Lancelot, J.-M. Idée, C. Laclédère, R. Santus, and C. Corot, "Effects of two dimeric iodinated contrast media on renal medullary blood perfusion and oxygenation in dogs," Investigative Radiology, vol. 37, no. 7, pp. 368-375, 2002.

[20] J. Ueda, A. Nygren, P. Hansell, and H. R. Ulfendahl, "Effect of intravenous contrast media on proximal and distal tubular hydrostatic pressure in the rat kidney," Acta Radiologica, vol. 34, pp. 83-87, 1993.

[21] M. Pedersen, T. H. Dissing, J. Morkenborg et al., "Validation of quantitative BOLD MRI measurements in kidney: application to unilateral ureteral obstruction," Kidney International, vol. 67, no. 6, pp. 2305-2312, 2005.

[22] P. V. Prasad, A. Priatna, K. Spokes, and F. H. Epstein, “Changes in intrarenal oxygenation as evaluated by BOLD MRI in a rat kidney model for radiocontrast nephropathy," Journal of Magnetic Resonance Imaging, vol. 13, no. 5, pp. 744-747, 2001.

[23] S. N. Heyman, M. Brezis, F. H. Epstein, K. Spokes, P. Silva, and S. Rosen, "Early renal medullary hypoxic injury from radiocontrast and indomethacin," Kidney International, vol. 40, no. 4, pp. 632-642, 1991.

[24] P. Liss, A. Nygren, U. Olsson, H. R. Ulfendahl, and U. Erikson, "Effects of contrast media and mannitol on renal medullary blood flow and red cell aggregation in the rat kidney," Kidney International, vol. 49, no. 5, pp. 1268-1275, 1996.

[25] E. Seeliger, B. Flemming, T. Wronski et al., "Viscosity of contrast media perturbs renal hemodynamics," Journal of the American Society of Nephrology, vol. 18, no. 11, pp. 2912-2920, 2007.

[26] X.-Z. Zhang and C. Baylis, "Endothelin mediates renal vascular memory of a transient rise in perfusion pressure due to NOS inhibition," The American Journal of Physiology-Renal Physiology, vol. 276, no. 4, pp. F629-F634, 1999.

[27] L. Dobrowolski, E. Kompanowska-Jezierska, A. Walkowska, and J. Sadowski, "Sodium intake determines the role of adenosine A2 receptors in control of renal medullary perfusion in the rat," Nephrology Dialysis Transplantation, vol. 22, no. 10, pp. 2805-2809, 2007.

[28] G. Deray, "Contrast-associated nephropathy_old clinical problem and new therapeutic perspectives," Nephrology Dialysis Transplantation, vol. 13, no. 11, pp. 2976-2977, 1998. 


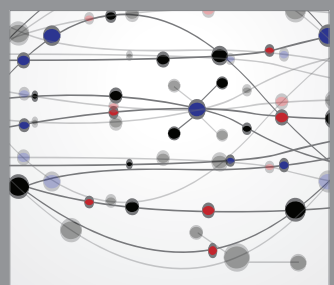

The Scientific World Journal
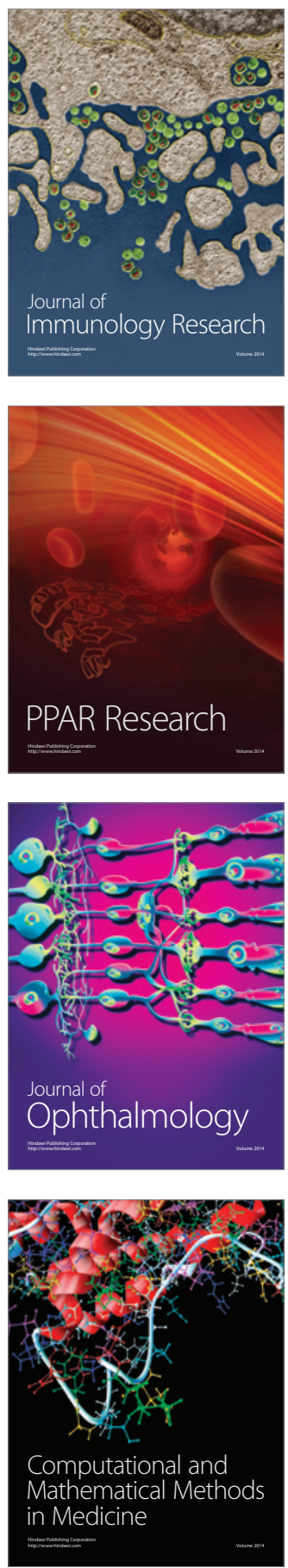

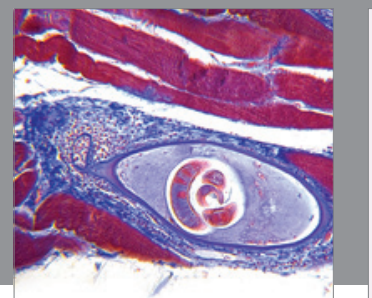

Gastroenterology

Research and Practice
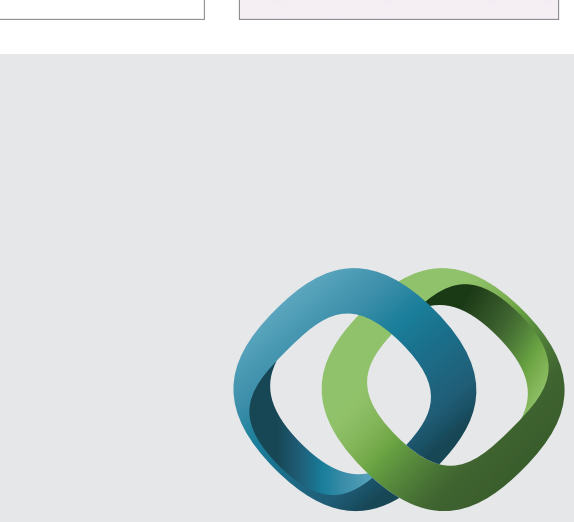

\section{Hindawi}

Submit your manuscripts at

http://www.hindawi.com
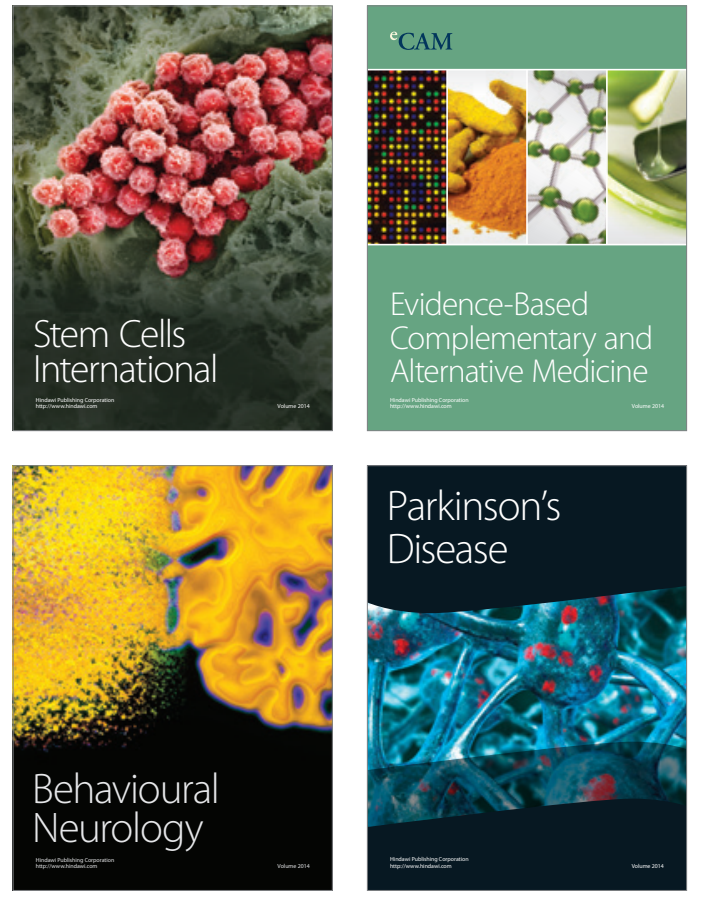
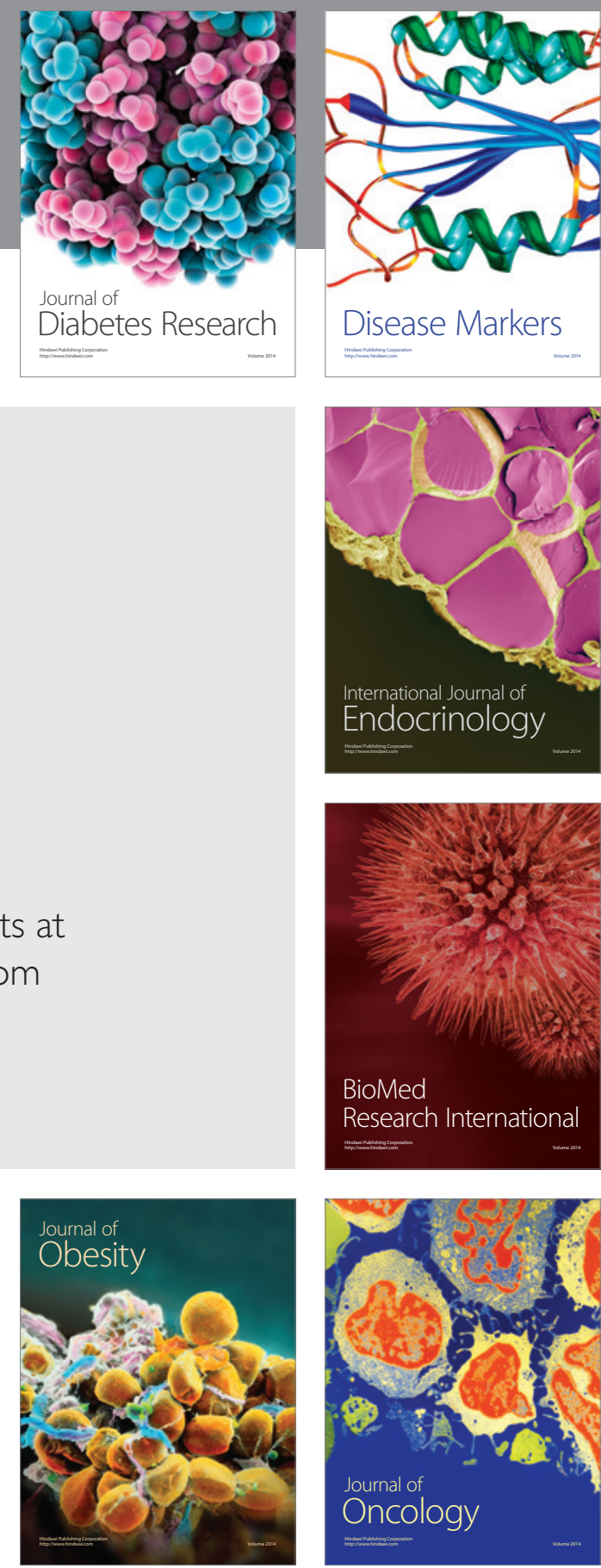

Disease Markers
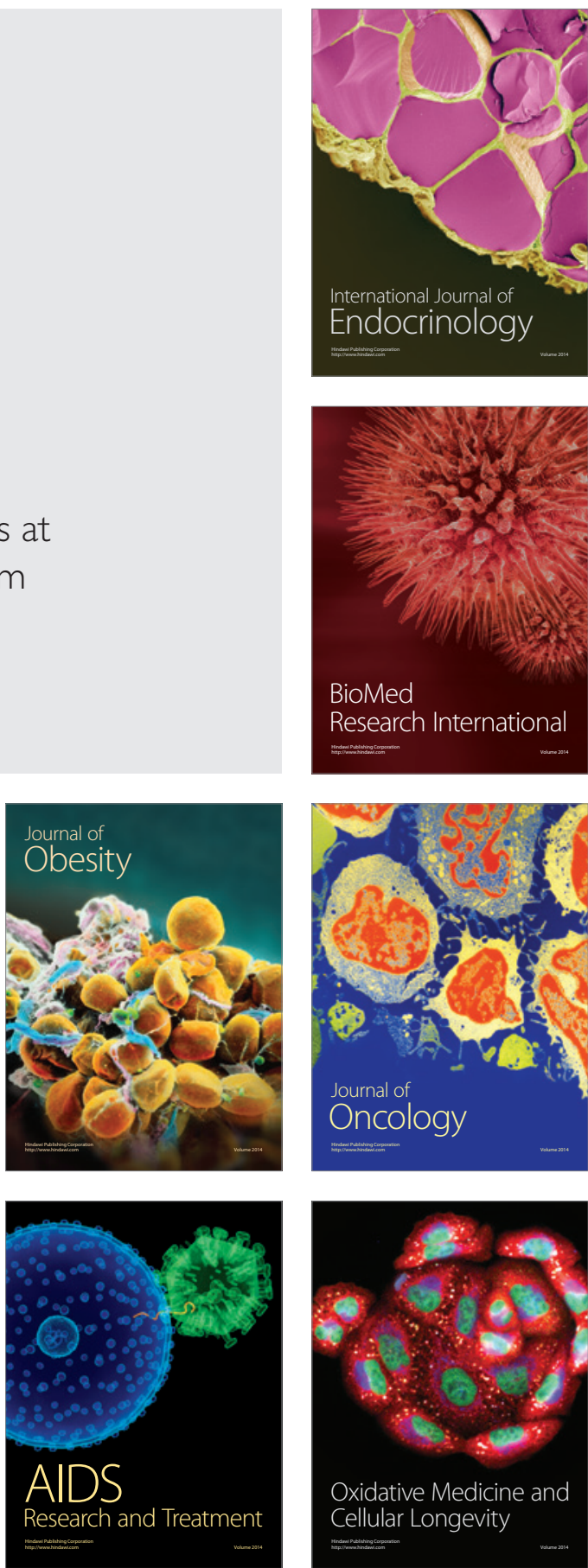